\begin{tabular}{|c|l|}
\hline Title & $\begin{array}{l}\text { A nalysis of the Pancharatnam-Berry phase of vector vortex states using the Hamiltonian based on the Maxwell- } \\
\text { Schrödinger equation }\end{array}$ \\
\hline Author(s) & Suzuki, Masato; Y amane, Keisaku; Oka, Kazuhiko; Toda, Y asunori; Morita, Ryuji \\
\hline Citation & $\begin{array}{l}\text { Physical Review A, 94(4), 043851-1-043851-7 } \\
\text { https://doi.org/10.1103/PhysRevA.94.043851 }\end{array}$ \\
\hline Issue Date & 2016-10-26 \\
\hline Doc URL & http://hdl.handle.net/2115/63476 \\
\hline Rights & O2016 A merican Physical Society \\
\hline Type & article \\
\hline File Information & PhysRevA.94.pdf \\
\hline
\end{tabular}

Instructions for use 


\title{
Analysis of the Pancharatnam-Berry phase of vector vortex states using the Hamiltonian based on the Maxwell-Schrödinger equation
}

\author{
Masato Suzuki, Keisaku Yamane, Kazuhiko Oka, Yasunori Toda, and Ryuji Morita* \\ Department of Applied Physics, Hokkaido University, Kita-13, Nishi-8, Kita-ku, Sapporo 060-8628, Japan
}

(Received 9 February 2016; published 26 October 2016)

\begin{abstract}
We derived the Berry connection of vector vortex states (VVSs) from the "true" Hamiltonian obtained through the Maxwell-Schrödinger equation for an inhomogeneous anisotropic (IA) medium, and we experimentally demonstrated measurement of the corresponding Pancharatnam-Berry (PB) geometrical phase of VVSs. The PB phase (PBP) of VVSs can be divided into two phases: homogeneous and inhomogeneous PBPs. Homogeneous and inhomogeneous PBPs are related to the conventional PBP and the spatially dependent geometric phase given by an IA medium such as a polarization converter, respectively. We theoretically detected that inhomogeneous PBP accumulation originates from the gauge dependence of the index of the hybrid-order Poincare sphere, which provides an alternate method for understanding optical spin-orbital angular momentum conversion. The homogeneous PBP, which is explicitly observed, has implications for quantum-state manipulation and information processing.
\end{abstract}

DOI: 10.1103/PhysRevA.94.043851

\section{INTRODUCTION}

The Pancharatnam-Berry phase (PBP) is a geometrical phase [1] associated with polarization of light [2]. The PBP has been experimentally observed using a homogeneously distributed polarization light state [3]. Recently, using spinorbit converters [4], Milione et al. [5] conducted a pioneering exploration of PBPs for vector vortex states (VVSs). Here, the VVSs, having attracted attention for many applications [6], are light states having both inhomogeneous phase and polarization distributions [7]. On a higher-order Poincaré sphere, which is the space of higher-order Stokes parameters [8], Milione et al. show pathways made by the spin-orbit converters.

A polarization converter to generate a vector vortex beam can make a path from a state on one to a state on another higherorder Poincaré sphere [9], whose process can be described on a hybrid-order Poincaré sphere (HOPS) [10]. Yi et al. proposed not only the HOPS but also the PBP for the HOPS, which are acquired similarly to the method of Milione et al. [5].

Milione et al. theoretically and experimentally investigated the PBP induced by spin-orbit converters composed of halfwave plates and pairs of cylindrical lenses. However, the PBP discussed by them does not always include the PBP induced by a sequence of inhomogeneous anisotropic (IA) media, which are another spin-orbit converters, such as space-variant wave plates (SVWPs) [11] or $q$ plates [12]. The transition of a state of a VVS in IA medium can be described on the HOPS, and we can acquire the PBP when the Hamiltonian for the IA medium is obtained. However, it is difficult to justify the Hamiltonian for an optical system [13]. Although Berry has shown the procedure to acquire the PBP for a uniformly polarized light state [14], the Hamiltonian is just the density matrix of the circularly polarized states, which does not provide an appropriate equation of motion for wave plates. Therefore, it is essential to acquire the true PBP from the true Hamiltonian describing an IA medium such as a SVWP or a $q$ plate, which is a typical spin-orbit converter.

\footnotetext{
*morita@eng.hokudai.ac.jp
}

In the present paper, in order to accurately discuss the PBP for VVSs in the right way, we will reestablish the Berry connections of VVSs on the HOPS. We first acquire the "true" Hamiltonian of a SVWPs as an extension of Refs. [15], which acquire the Hamiltonian of homogeneous birefringent media from the Maxwell-Schrödinger equation. Furthermore, we will experimentally measure the PBP for VVSs and demonstrate it to be a "true" PBP given by IA media.

\section{THEORETICAL FORMULATION}

\section{A. Hybrid-order Stokes parameters}

We use bra-ket notation to describe a VVS:

$$
\begin{aligned}
|\psi\rangle & =\frac{\psi_{+, l} e^{-i l_{+} \phi}}{\sqrt{2}}\left(\begin{array}{l}
1 \\
i
\end{array}\right)+\frac{\psi_{-, l} e^{i l_{-} \phi}}{\sqrt{2}}\left(\begin{array}{r}
1 \\
-i
\end{array}\right) \\
& =\frac{e^{i l^{\prime} \phi}}{\sqrt{2}}\left[\psi_{+, l} e^{-i l \phi}\left(\begin{array}{l}
1 \\
i
\end{array}\right)+\psi_{-, l} e^{i l \phi}\left(\begin{array}{r}
1 \\
-i
\end{array}\right)\right],
\end{aligned}
$$

where $\phi$ is the azimuthal angle, $\psi_{ \pm, l}$ are amplitude functions, $l_{+}$and $l_{-}$indicate topological charges of the left- and right-circularly polarized states, respectively, and $l^{\prime}\left[=\left(-l_{+}+\right.\right.$ $\left.\left.l_{-}\right) / 2\right]$ and $l\left[=\left(l_{+}+l_{-}\right) / 2\right]$ are azimuthal indices. We require the inner product of the vector to be unity $\left(\left|\psi_{+, l}\right|^{2}+\left|\psi_{-, l}\right|^{2}=\right.$ 1). By definition, the hybrid-order Stokes parameters are described as

$$
\boldsymbol{S}_{-l_{+}, l_{-}}=\left[\begin{array}{c}
1 \\
S_{1}^{-l_{+}, l_{-}} \\
S_{2}^{-l_{+}, l_{-}} \\
S_{3}^{-l_{+}, l_{-}}
\end{array}\right]=\langle\phi|\boldsymbol{\sigma}| \phi\rangle,
$$

where $\sigma=\left[\sigma_{0}(\equiv \hat{1}), \sigma_{1}, \sigma_{2}, \sigma_{3}\right]^{\mathrm{T}}$ are the Pauli spin matrices [16], and $|\phi\rangle$ represents $\left(\psi_{+, l}, \psi_{-, l}\right)^{\mathrm{T}}$. Since $S_{i}^{-l_{+}, l_{-}}=S_{i}^{l}(i=$ $1,2)$ and $S_{3}^{-l_{+}, l_{-}} \equiv S_{3}$, we hereafter express $\boldsymbol{S}_{-l_{+}, l_{-}}$as $\boldsymbol{S}_{l}$, and $\tilde{\boldsymbol{S}}_{l}$ as $\left[S_{1}^{l}, S_{2}^{l}, S_{3}\right]^{\mathrm{T}}$. If $l$ is an integer, $\boldsymbol{S}_{l}$ represents the higher-order Stokes parameters. 


\section{B. Hamiltonian for space-variant wave plates}

Following Refs. [15], the Maxwell-Schrödinger equation for an IA medium, whose transverse dielectric tensor is described by $\hat{\epsilon}_{\perp}=\left(\epsilon_{i j}\right)(i, j=x, y)$, is

$$
2 i \sqrt{\epsilon} k^{-1} \partial_{z}|\phi\rangle=\left(\epsilon \hat{1}-T_{l} \hat{\epsilon}_{\perp} T_{l}^{\dagger}\right)|\phi\rangle,
$$

where $\epsilon$ is a dielectric value, $k$ is a wave number, and

$$
T_{l}=\left(\begin{array}{cc}
e^{i l \phi} & -i e^{i l \phi} \\
e^{-i l \phi} & i e^{-i l \phi}
\end{array}\right)
$$

is a transform matrix from the $x, y$-basis representation $|\psi\rangle$ to the circularly polarized optical vortex basis representation $|\phi\rangle$ (see Appendix A). Here, the Hamiltonian $\mathcal{H}_{l}$ is given by $\epsilon \hat{1}-T_{l} \hat{\epsilon}_{\perp} T_{l}^{\dagger}$.

The transverse relative permittivity tensor of a $q=l, \alpha_{0}=$ $l \bar{\alpha}$ wave plate is described using a rotational matrix $R_{\theta}$ :

$$
\hat{\epsilon}_{\perp}=R_{l(\phi+\bar{\alpha})}\left(\begin{array}{cc}
\epsilon_{\mathrm{o}} & 0 \\
0 & \epsilon_{\mathrm{e}}
\end{array}\right) R_{-l(\phi+\bar{\alpha})},
$$

where $\epsilon_{\mathrm{o}, \mathrm{e}}$ are the extraordinary and the ordinary dielectric constants, respectively. Note that the definitions of $q$ and $\alpha_{0}$ are given in Ref. [12], and when $l=0, l \bar{\alpha}$ is replaced with $\bar{\alpha}$. Hence, the Hamiltonian is calculated to be $\mathcal{H}_{l}=$ $\epsilon_{-}\left[\cos (l \bar{\alpha}) \sigma_{1}+\sin (l \bar{\alpha}) \sigma_{2}\right]$, where $\epsilon_{ \pm}=\left( \pm \epsilon_{\mathrm{o}}+\epsilon_{\mathrm{e}}\right) / 2$ and $\epsilon=$ $\epsilon_{+}$. The evolution equation for $\tilde{\boldsymbol{S}}_{l}$ is given [15] by

$$
\frac{d \tilde{\boldsymbol{S}}_{l}}{d \delta}=\tilde{\boldsymbol{S}}_{l} \times\left[\begin{array}{c}
-\cos (2 l \bar{\alpha}) \\
-\sin (2 l \bar{\alpha}) \\
0
\end{array}\right],
$$

where $\delta=\delta(z)=k \epsilon_{-} z / \sqrt{\epsilon_{+}}$is the retardance phase. This equation supplies the true path made by a SVWPs because Eq. (5) represents precession on the HOPS.

We can easily obtain the dynamical phase by following the Berry's discussion [16]. The dynamical phase $\gamma_{\text {dynamical }}$ is given by

$$
\gamma_{\text {dynamical }}=-\frac{k}{2 \sqrt{\epsilon_{+}}} \int_{0}^{z} \lambda_{ \pm}\left(\tilde{\boldsymbol{S}}_{l}\right) d z,
$$

where $\lambda_{ \pm}$are the eigenvalues of the following eigenvalue equation:

$$
\mathcal{H}_{l}\left|\psi_{ \pm}\left(\tilde{\boldsymbol{S}}_{l}\right)\right\rangle=\lambda_{ \pm}\left(\tilde{\boldsymbol{S}}_{l}\right)\left|\psi_{ \pm}\left(\tilde{\boldsymbol{S}}_{l}\right)\right\rangle
$$

Since $\lambda_{ \pm}= \pm \epsilon_{-}$, the dynamical phases $\gamma_{\text {dynamical }}$ for $\left|\psi_{ \pm}\left(\tilde{\boldsymbol{S}}_{l}\right)\right\rangle$ are given by $\pm \delta / 2$, respectively. Hence, the dynamical phase is interpreted as the retardance phase.

\section{Berry connection of vector vortex states}

We now obtain the PBP of VVSs through the Berry connection. Since $\mathcal{H}_{l}$ is equivalent to a spin-1/2 system Hamiltonian, the Berry connection is expressed by

$$
\boldsymbol{A}\left(\tilde{\boldsymbol{S}}_{l}\right)=-\boldsymbol{e}_{\zeta_{l}}\left(2 R_{l}\right)^{-1} \tan \frac{\xi_{l}}{2}+\nabla_{\tilde{\boldsymbol{S}}_{l}} \Phi\left(\tilde{\boldsymbol{S}}_{l}\right)
$$

where $\Phi\left(\tilde{\boldsymbol{S}}_{l}\right)$ is a scalar potential [17]. Here, we used the spherical coordinates $\tilde{\boldsymbol{S}}_{l}=R_{l} \boldsymbol{e}_{R_{l}}+\xi_{l} \boldsymbol{e}_{\xi_{l}}+\zeta_{l} \boldsymbol{e}_{\zeta_{l}}$, where $R_{l}, \xi_{l}$, and $\zeta_{l}$ are the radial distance, polar angle, and azimuthal angle in the $l$ th HOPS, and $\boldsymbol{e}_{i}\left(i=R_{l}, \xi_{l}, \zeta_{l}\right)$ represents the unit vector for the $i$ axis. We define the PBP of VVSs through the Berry connection as

$$
\gamma_{\text {Berry }}=\sum_{i=0}^{i_{\max }} \int_{\tilde{\boldsymbol{S}}_{l_{i}}^{\mathrm{S}}}^{\tilde{\boldsymbol{S}}_{l_{i}}^{\mathrm{F}}} \boldsymbol{A}\left(\tilde{\boldsymbol{S}}_{l}\right) \cdot d \tilde{\boldsymbol{S}}_{l_{i}},
$$

where we require a closed loop in the general meaning. $\tilde{\boldsymbol{S}}_{l_{0}}^{\mathrm{S}}=$ $\tilde{\boldsymbol{S}}_{l_{\max }}^{\mathrm{F}}$ and $\tilde{\boldsymbol{S}}_{l_{i}}^{\mathrm{S}}=\tilde{\boldsymbol{S}}_{l_{i-1}}^{\mathrm{F}}$, but $l_{0} \neq l_{\max }$ and $l_{i} \neq l_{i-1}$ are accepted if $\tilde{S}_{l_{i}}^{\mathrm{S}}=[0,0, \pm 1]^{\mathrm{T}}$.

\section{Gauge dependence on $l$}

In general, the Berry phase is gauge invariant. However, we allow the jump from one HOPS to another HOPS at the north and the south poles on the HOPS, so the BP phase may be gauge variant when the gauge depends on $l$. The general solution of Eq. (3) is

$$
|\psi\rangle=e^{i\left(l^{\prime} \phi-\delta(z) / 2\right)} R_{l(\phi+\bar{\alpha})}\left(\begin{array}{c}
E_{x}^{0} \\
E_{y}^{0} e^{i \delta(z)}
\end{array}\right),
$$

where $e^{i l \phi} R_{l(\phi+\bar{\alpha})}\left(E_{x}^{0}, E_{y}^{0}\right)^{\mathrm{T}}$ is the initial state of $|\psi\rangle$ at $z=0$. An overall phase $\Phi_{\text {overall }}$ of Eq. (10) is calculated to be (see Appendix B)

$$
\begin{aligned}
\Phi_{\text {overall }}= & l^{\prime} \phi-\arg \left[E_{x}^{0} e^{-i \delta / 2} \cos l(\phi+\bar{\alpha})\right. \\
& \left.-E_{y}^{0} e^{i \delta / 2} \sin l(\phi+\bar{\alpha})\right] .
\end{aligned}
$$

Because the overall phase does not depend on an initial state (see Appendix B), we set the initial state to be the north pole of the $l$ th HOPS $\left(E_{x}^{0}=1, E_{y}^{0}=i\right)$. In that case, $\left(R_{l}, \xi_{l}, \zeta_{l}\right)=$ $(1, \delta, 2 l \bar{\alpha}-\pi / 2)$; thus, the overall phase is rewritten by

$$
\Phi_{\text {overall }}\left(\tilde{\mathbf{S}}_{l}\right)=l^{\prime} \phi+\arctan \frac{\tan \left(\pi / 4+\xi_{l} / 2\right)}{\tan \left(l \phi+\zeta_{l} / 2\right)} .
$$

Since Eq. (12) depends on $l$, which results in the gauge-variant PBP, we express the scalar potential as

$$
\Phi\left(\tilde{\boldsymbol{S}}_{l}\right)=\Phi_{\text {overall }}\left(\tilde{\mathbf{S}}_{l}\right)+\Phi_{\text {indep }}\left(\tilde{\mathbf{S}}_{l}\right),
$$

where $\Phi_{\text {indep }}$ is an arbitrary function independent of $l$. Thus, Eq. (8) can be divided into terms independent of $l$, $\left[-\boldsymbol{e}_{\zeta_{l}}\left(2 R_{l}\right)^{-1} \tan \left(\xi_{l} / 2\right)+\nabla_{\tilde{S}_{l}} \Phi_{\text {indep }}\left(\tilde{\mathbf{S}}_{l}\right)\right]$, and the term dependent on $l,\left[\nabla_{\tilde{S}_{l}} \Phi_{\text {overall }}\left(\tilde{\mathbf{S}}_{l}\right)\right]$. If the trajectory satisfies the closed loop in the general meaning, the PBP is described by

$$
\begin{aligned}
\gamma_{\text {Berry }} & =-\frac{\Omega}{2}+\sum_{i=0}^{i_{\max }}\left[\Phi_{\text {overall }}\left(\tilde{\mathbf{S}}_{l}\right)\right]_{\tilde{S}_{l_{i}}^{\mathrm{S}}}^{\tilde{\boldsymbol{S}}_{l_{i}}^{\mathrm{F}}} \\
& =-\frac{\Omega}{2}-\frac{\phi}{2} \sum_{i=0}^{i_{\max }}\left[\left(\cos \xi_{l_{i}}^{\mathrm{F}}-\cos \xi_{l_{i}}^{\mathrm{S}}\right) l_{i}\right],
\end{aligned}
$$

where $\Omega$ is the area subtended by the closed loop [Fig. 1(a)]. Hence, when the closed loop is on one HOPS, the PBP is not gauge dependent, but when it travels between HOPSs, the PBP is gauge dependent. The former and the latter terms of Eq. (14) are homogeneous and inhomogeneous parts of the PBP, respectively. From the requirement of the closed loop, the inhomogeneous part is quantized by $\phi$, which is illustrated by a "ladder chart" [Fig. 1(b)]. This is one of the key results of this paper. 
(a)

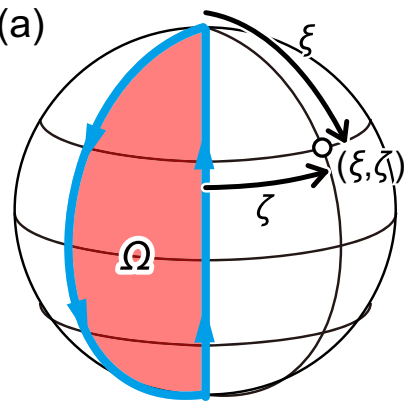

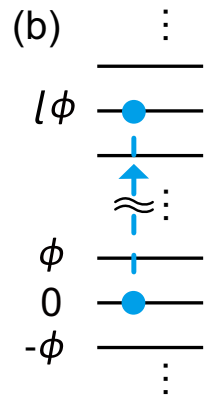

FIG. 1. (a) A conceptual diagram of the area $\Omega$ subtended by the closed loop on the HOPS and definition of the angles $\xi$ and $\zeta$. (b) A ladder chart.

\section{EXPERIMENT}

\section{A. Experimental setup}

Here, we experimentally measured the PBP for VVSs through interferometry analogous to the measurement of the Aharonov-Bohm effect $[14,18]$. Figure 2 shows the experimental setup. The light source that we used in this experiment was a Ti:sapphire laser amplifier (center wavelength $800 \mathrm{~nm}$; bandwidth $\sim 40 \mathrm{~nm}$; pulse duration $\sim 25 \mathrm{fs}$ ). The attenuated pulse from the laser amplifier passed through a bandpass filter (BPF; center wavelength, $800 \mathrm{~nm}$; bandwidth $10 \mathrm{~nm}$ ), lengthening its pulse duration to $\sim 120 \mathrm{fs}$ ( $\sim 40$ cycles). We conducted this experiment by use of many-cycle femtosecond pulses [19]. After the BPF, a spatial light modulator (SLM) system shaped the spatial intensity profile of the pulse into a Gaussian profile. The $x$-polarized (or horizontally polarized) pulse was branched into two beams at a beam splitter (BS1). In the upper branch, the polarization state of light travels on the HOPSs; in the lower branch, light is directed into the delay line as a reference pulse beam (Fig. 2). Here, BS1 and a second beam splitter (BS2) form a Mach-Zehnder

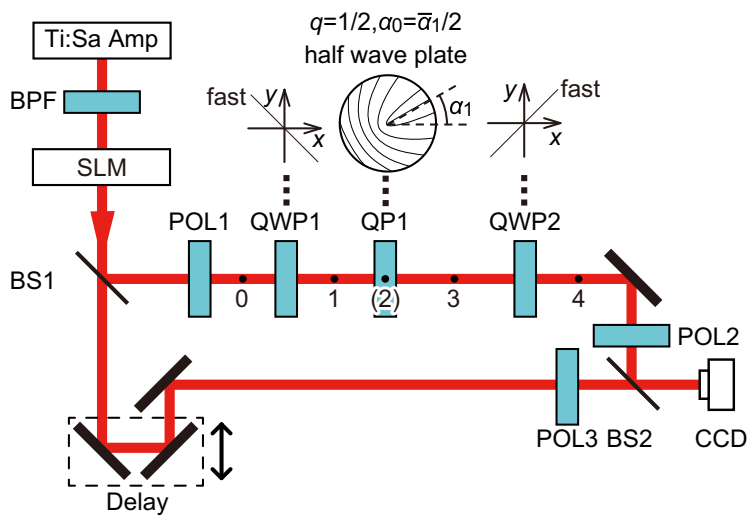

FIG. 2. The experimental setup for measuring PBP for VVSs, where BPF is a bandpass filter (center wavelength $800 \mathrm{~nm}$; bandwidth $10 \mathrm{~nm}$ ); SLM is a liquid crystal on a silicon spatial light modulator; BS1,2 are 50:50 nonpolarizing beam splitters for ultrafast optics (Thorlabs UFBS5050); POL1,2,3 are polarizers; QWP1,2 are quarter-wave plates; QP1 is a $q=1 / 2, \alpha_{0}=\bar{\alpha}_{1} / 2$ half-wave plate (Photonic Lattice SWP-808); Delay is a delay stage; and CCD is a charge-coupled device camera. The points are numbered in order to distinguish the intermediate states. Point 2 is located inside QP1.
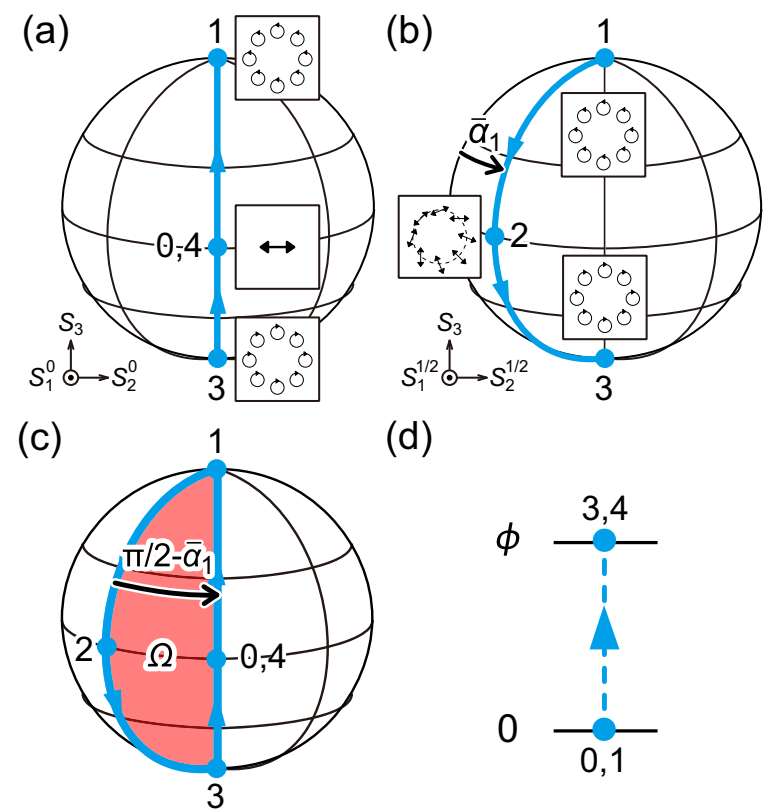

(d)

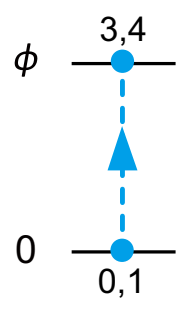

FIG. 3. Paths on the HOPSs for (a) $l=0$ and (b) $l=1 / 2$. (c) Superposition of all trajectories on all HOPSs [(a) and (b)]. Here, $\Omega$ is the solid angle of the circuit drawn by the trajectories. State 2 is expedientially illustrated to be $\tilde{\boldsymbol{S}}_{1 / 2}=\left[\sin \left(\bar{\alpha}_{1}-\pi / 4\right),-\cos \left(\bar{\alpha}_{1}-\pi / 4\right), 0\right]^{\mathrm{T}}$. (d) A ladder chart.

interferometer. In the upper branch, a polarizer (POL1) purified the $x$-polarized state. A quarter-wave plate (QWP1) with the fast axis at $3 \pi / 4 \mathrm{rad}$ to the $x$ axis converted the polarization state into left circularly polarized. After that, the pulse passed through a $q=1 / 2, \alpha_{0}=\bar{\alpha}_{1} / 2$ half-wave plate (QP1, $\bar{\alpha}_{1}$ is a rotation angle depicted in Fig. 2), and the pulse went through a quarter-wave plate with the fast axis at $\pi / 4$ rad to the $x$ axis. Although the spatial profile is converted into a "point vortex" [20] or a hypergeometric-Gaussian mode [21] by a polarization converter QP1, the spatial intensity profile after QP1 was returned to a Gaussian profile on the charge-coupled camera (CCD) due to the pair of relay lenses. After the polarizers with polarization axes along the $x$ axis (POL2 and POL3) purified the $x$-polarized states, a beam splitter (BS2) combined the upper and lower beams collinearly and coherently. The delay time was set so that the contrast of the interference was sufficiently high. The spatial interference pattern was captured by CCD.

Figures 3(a) and 3(b) depict the paths of the VVS on the HOPSs. By merging all trajectories into one sphere as shown in Fig. 3(c), these paths can form a closed contour, which satisfies the requirement of a "closed" loop. From Eq. (14), the PBP is calculated to be $-\Omega / 2+\phi=\bar{\alpha}_{1}-\pi / 2+\phi$. Early studies [11] have mentioned the observation of the inhomogeneous PBP term $\phi$ through a $q=1 / 2$ half-wave plate, which is known as the space-variant Pancharatnam-Berry phase. However, the uniform PBP term $\bar{\alpha}_{1}-\pi / 2$ has not been explicitly observed. Figure $3(\mathrm{~d})$ is a ladder chart describing the change in the inhomogeneous part of the PBP.

\section{B. Results}

Figure 4 shows the experimental results. To explore the PBP for VVSs, we measured the intensity of the interference 
(a)
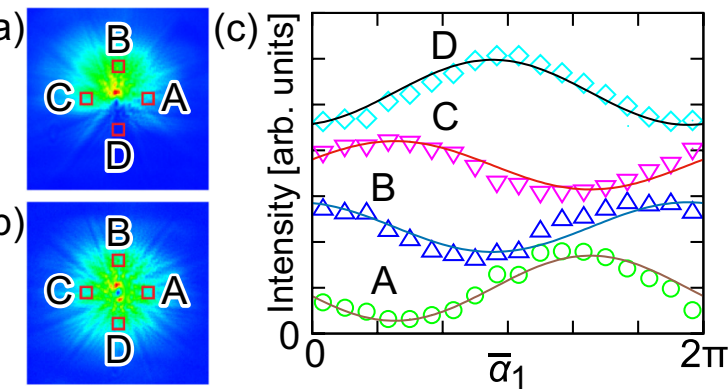

FIG. 4. Experimental results. (a, b) Intensity patterns acquired with a CCD camera with and without the reference pulse beam, respectively. The beam size of the reference pulse beam is large enough to cover the whole original pulse beam (b). In contrast to the intensity pattern of the original pulse beam (b), the interference pattern (a) is nonuniform in the azimuthal direction, which shows that there is a nonuniform phase distribution in the azimuthal direction. Red rectangles in (a) and (b) represent the average areas. (c) Intensity variation by changing the rotation angle $\bar{\alpha}_{1}$. Curves in (c) are the fitted lines proportional to $\cos \left(\gamma_{\text {Berry }}+\delta_{\text {pd }}\right)+$ const.

by rotating QP1. The measured intensity patterns are shown in Fig. 4(a). The beam center was estimated using the singular point on the intensity pattern without the reference pulses [Fig. 4(b)]. Areas A, B, C, and D correspond to $\phi=0, \pi / 2, \pi$, and $3 \pi / 2$, respectively. The intensity is proportional to the function of $\cos \left(\gamma_{\text {Berry }}+\delta_{\mathrm{pd}}\right)+$ const., where $\delta_{\mathrm{pd}}$ is the phase difference owing to delay and was experimentally evaluated to be $3.38 \mathrm{rad}$. The dynamical phase is a constant value in the beam cross section and is almost canceled by the interference measurements. The obtained data in Fig. 4(c) are consistent with the above function; thus we have successfully observed the PBP for VVSs, including not only the inhomogeneous part but also the homogeneous part.

\section{DISCUSSION}

The experimental results in our setup show that the inhomogeneous part of the PBP for VVSs is gauge dependent on $l$. If we replace QP1 (a $q=1 / 2$ half-wave plate) with an ordinary $(q=0)$ half-wave plate, the experimental setup is essentially the same as the well-known homogeneous PBP measurement setup [3], and the trajectories on all HOPSs correspond to Fig. 3(c). The difference in the two setups is whether there are jumps between different HOPSs at the poles or not. The interference pattern varying in the azimuthal direction in Fig. 4(a), which cannot be observed in the conventional homogeneous PBP measurement setup, shows that the inhomogeneous part of the PBP for VVSs depends on jumps between different HOPSs at poles, indicating the gauge dependency of the PBP.

The PBP for VVSs is composed of the homogeneous and inhomogeneous PBP [Eq. (14)]. While the former is the same as the PBP for homogeneously polarized states, the latter is unique for VVSs. Thus, we write the general formula of the PBP for VVSs as

$$
\gamma_{\text {Berry }}(\phi)=\gamma_{\text {Berry,H }}+\gamma_{\text {Berry,I }}(\phi),
$$

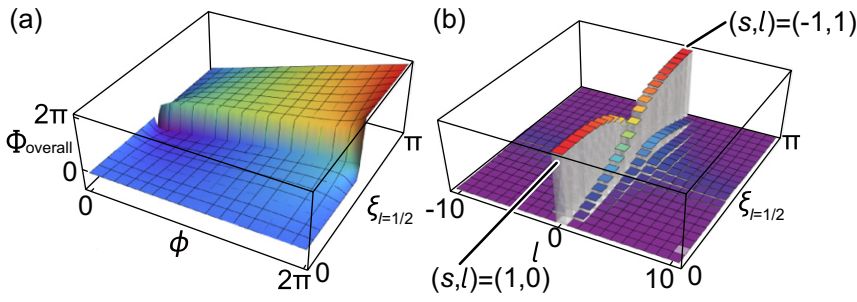

FIG. 5. (a) Distribution of $\Phi_{\text {overall }}\left(\phi, \xi_{l}\right)$ and (b) its topological charge power spectrum when $l=1 / 2, \theta_{0}=\pi / 2$, and $l^{\prime}=1 / 2$.

where $\gamma_{\text {Berry,H }}=-\Omega / 2$ and $\gamma_{\text {Berry, }}(\phi)$, respectively, stand for homogeneous and inhomogeneous PBPs. The former is illustrated by the area subtended by the closed contour on superposed HOPS [Fig. 1(a)]. The latter can be described by the ladder chart in Fig. 1(b), where the intermediate states between the initial and the final states are complicated, when the trajectory satisfies our requirement of the "closed" loop.

From Eq. (12), in a $q=l$ wave plate, the phase ramp of the inhomogeneous PBP around the beam axis is homogeneous only at the north pole $\left(\xi_{l}=0\right)$, the south pole $\left(\xi_{l}=\pi\right)$, and the equator $\left(\xi_{l}=\pi / 2\right)$ of the $l$ th HOPS, but the distribution along the $\phi$ axis of the inhomogeneous PBP is generally complicated. Figure 5 shows the inhomogeneous $\operatorname{PBP} \Phi_{\text {overall }}\left(\phi, \xi_{l}\right)$ and its topological charge distribution (the Fourier power spectrum of $\exp \left[i \Phi_{\text {overall }}\left(\phi, \xi_{l}\right)\right]$ on the $\phi$ axis) for $q=1 / 2, \alpha=\pi / 2$ halfwave plates. The transition of VVSs in SVWPs is interpreted as the optical spin-to-orbital angular momentum conversion from $(s, l)=\left( \pm 1, l^{\prime} \mp l\right)$ to $\left(\mp 1, l^{\prime} \pm l\right)(s$ is the spin angular momentum in units of $\hbar$ ), which has been conventionally regarded as energy conversion between the left-circularly polarized $\left(s=1, \xi_{l}=0\right)$ and right-circularly polarized $\left(s=-1, \xi_{l}=\pi\right)$ states $[12,22]$. We further introduce another interpretation of this phenomena: the transition of VVSs in SVWPs is the adiabatic change of the topological charge spectrum of the inhomogeneous PBP, as shown in Fig. 5(b).

\section{CONCLUSION}

We have introduced the Berry connections of VVSs from the Maxwell-Schrödinger equation, and we experimentally verified the PBP obtained from the Berry connections. In contrast to earlier studies, our PBP of VVSs describes an adiabatic change of a VVS on a HOPS. We have found that the PBP can be divided into two phases. One phase is the homogeneous $\operatorname{PBP} \gamma_{\text {Berry,H }}$, which is essentially the same mathematics as the conventional PBP and is explicitly observed. The other phase is the inhomogeneous PBP $\gamma_{\text {Berry,I }}(\phi)$, which is ascribed to the gauge dependence of $l$. We have theoretically detected the adiabatic change of the inhomogeneous PB phase and its topological charge spectrum in polarization converters, which provide another aspect of the optical spin-orbital angular momentum conversion. This research was conducted only with $q$ retarders, which confine motions on the HOPS. Observations of the PBPs using various optical effects such as Faraday rotation are desired.

Adiabatic manipulation of the quantum coherence of spinor Bose-Einstein condensates (BECs) through the stimulated Raman adiabatic passage (STIRAP) process using optical 
vortex pulses as pump pulses can be interpreted as a Raman $q$-wave plate for spinor BECs [23]. Therefore, the PBP for VVSs has implications for spinor BECs and related other quantum systems. In particular, the homogeneous PBP can be applied to quantum phase gates and precise phase manipulation of macroscopic quantum states.

\section{ACKNOWLEDGMENTS}

This work was partially supported by JSPS KAKENHI Grants No. JP26286056, No. JP15J00038, and No. JP16H06506, and CREST, JST.

\section{APPENDIX A: DERIVATIONS FOR THE MAXWELL-SCHRÖDINGER EQUATION IN THE CIRCULARLY POLARIZED OPTICAL VORTEX BASIS}

We suppose that the relative permittivity tensor $\hat{\epsilon}$ and the electric field vector $\boldsymbol{E}(\boldsymbol{r}, t)$ are, respectively, described by

$$
\begin{gathered}
\hat{\epsilon}=\left(\begin{array}{ccc}
\epsilon_{x x} & \epsilon_{x y} & 0 \\
\epsilon_{y x} & \epsilon_{y y} & 0 \\
0 & 0 & \epsilon_{z z}
\end{array}\right), \\
\boldsymbol{E}(\boldsymbol{r}, t)=\tilde{\boldsymbol{E}}(\boldsymbol{r}) e^{-i \omega t} .
\end{gathered}
$$

From the Maxwell equations, we derive the following two equations [15]:

$$
\begin{gathered}
\nabla^{2} \tilde{\boldsymbol{E}}+\hat{\epsilon} k^{2} \tilde{\boldsymbol{E}}=\nabla(\nabla \cdot \tilde{\boldsymbol{E}}), \\
\nabla \cdot(\hat{\epsilon} \tilde{\boldsymbol{E}})=0
\end{gathered}
$$

where the dispersion relation in vacuum, $k=\omega / \mathrm{c} \equiv \omega \sqrt{\epsilon_{0} \mu_{0}}$, is applied. Here, c, $\epsilon_{0}$, and $\mu_{0}$ are the velocity of light in vacuum, the permittivity of vacuum, and the permeability of vacuum, respectively.

We express the transverse electric field $\tilde{\boldsymbol{E}}$ as

$$
\begin{aligned}
\tilde{\boldsymbol{E}}_{\perp}(\boldsymbol{r}) & =\left(\begin{array}{c}
\tilde{E}_{x}(\boldsymbol{r}) \\
\tilde{E}_{y}(\boldsymbol{r})
\end{array}\right)=e^{i k \sqrt{\epsilon} z} f(r, z) T^{\dagger}|\phi\rangle \\
& \equiv e^{i k \sqrt{\epsilon} z} f(r, z) \frac{1}{\sqrt{2}}\left(\begin{array}{cc}
e^{-i l \phi} & e^{i l \phi} \\
i e^{-i l \phi} & -i e^{i l \phi}
\end{array}\right)\left(\begin{array}{c}
\psi_{+, l}(z) \\
\psi_{-, l}(z)
\end{array}\right),
\end{aligned}
$$

where $\epsilon=\left(\epsilon_{\mathrm{o}}+\epsilon_{\mathrm{e}}\right) / 2$. Here, we require that $f(r, z) T^{\dagger}$ satisfies the paraxial wave equation:

$$
\left(\nabla_{\perp}^{2}+2 i k \sqrt{\epsilon} \partial_{z}\right) f(r, z) T^{\dagger}=0 .
$$

From Eqs. (A1) and (A4), we derive

$$
\partial_{z} \tilde{E}_{z}=-\frac{1}{\epsilon_{z z}} \nabla_{\perp} \cdot\left(\hat{\epsilon}_{\perp} \tilde{\boldsymbol{E}}_{\perp}\right),
$$

and thus Eq. (A3) is transformed into

$$
\begin{aligned}
{\left[\left(\nabla_{\perp}^{2}\right.\right.} & \left.\left.+2 i k \sqrt{\epsilon} \partial_{z}\right) f(r, z) T^{\dagger}\right]|\phi\rangle+f(r, z) T^{\dagger}\left[\left(\nabla_{\perp}^{2}+2 i k \sqrt{\epsilon} \partial_{z}\right)\right. \\
& \left.-k^{2}\left(\epsilon T T^{\dagger}-T \hat{\epsilon}_{\perp} T^{\dagger}\right)\right]|\phi\rangle \\
= & \left.\nabla_{\perp}\left[\nabla_{\perp} \cdot\left(\hat{1}-\frac{\hat{\epsilon}_{\perp}}{\epsilon_{z z}}\right) f T^{\dagger}|\phi\rangle\right)\right] .
\end{aligned}
$$

Here, the first term of Eq. (A7) vanishes due to Eq. (A5).
Since $\nabla_{\perp}^{2}|\phi\rangle=\mathbf{0}$, we derive a simplified form of Eq. (A7):

$$
\begin{aligned}
& {\left[2 i k \sqrt{\epsilon} \partial_{z}-k^{2}\left(\epsilon \hat{1}-T \hat{\epsilon}_{\perp} T^{\dagger}\right)\right]|\phi\rangle} \\
& \quad=\frac{T}{f} \nabla_{\perp}\left[\nabla_{\perp} \cdot\left(\hat{1}-\frac{\hat{\epsilon}_{\perp}}{\epsilon_{z z}}\right) f T^{\dagger}|\phi\rangle\right] .
\end{aligned}
$$

Here, we discuss the right-hand side of Eq. (A8). If the medium is a $q=l, \alpha_{0}=l \bar{\alpha}$ retarder, the transverse dielectric tensor is described by

$$
\hat{\epsilon}_{\perp}=R_{l(\phi+\bar{\alpha})}\left(\begin{array}{cc}
\epsilon_{\mathrm{o}} & 0 \\
0 & \epsilon_{\mathrm{e}}
\end{array}\right) R_{-l(\phi+\bar{\alpha})},
$$

where $R_{\theta}$ is a rotation matrix

$$
R_{\theta}=\left(\begin{array}{cc}
\cos \theta & -\sin \theta \\
\sin \theta & \cos \theta
\end{array}\right)
$$

Thus, the right side is transformed into

$$
\begin{aligned}
\frac{T}{f} \nabla_{\perp} & {\left[\nabla_{\perp} \cdot\left(\hat{1}-\frac{\hat{\epsilon}_{\perp}}{\epsilon_{z z}}\right) f T^{\dagger}|\phi\rangle\right] } \\
= & \frac{1}{f}\left(1-\frac{\epsilon_{+}}{\epsilon_{z z}}\right)\left(\begin{array}{cc}
e^{i l \phi} \nabla_{\perp}^{2} & e^{i i \phi} \hat{l}_{-}^{2} \\
e^{-i l \phi} \hat{l}_{+}^{2} & e^{-i l \phi} \nabla_{\perp}^{2}
\end{array}\right) f\left(\begin{array}{c}
e^{-i l \phi} \psi_{+, l} \\
e^{i l \phi} \psi_{-, l}
\end{array}\right) \\
& +\frac{1}{f} \frac{\epsilon_{-}}{\epsilon_{z z}}\left(\begin{array}{cc}
e^{i l \phi} \hat{l}_{-}^{2} & e^{i l \phi} \nabla_{\perp}^{2} \\
e^{-i l \phi} \nabla_{\perp}^{2} & e^{-i l \phi} \hat{l}_{+}^{2}
\end{array}\right) f\left(\begin{array}{c}
e^{i l(\phi+2 \bar{\alpha})} \psi_{+, l} \\
e^{-i l(\phi+2 \bar{\alpha})} \psi_{-, l}
\end{array}\right),
\end{aligned}
$$

where $\hat{l}_{ \pm}=\partial_{x} \pm i \partial_{y}$ are ladder operators [24]. These terms are regarded as energy conversion terms between $\psi_{ \pm, l}$, namely, the optical spin-orbit coupling [25]. These phenomena are negligible when the collimated beam (or nearly collimated beam) is propagating in a birefringent media [26]. We therefore neglect these terms.

Finally, the Maxwell-Schrödinger equation in the circularly polarized optical vortex basis is acquired:

$$
\frac{2 i \sqrt{\epsilon}}{k} \partial_{z}|\phi\rangle=\left(\epsilon \hat{1}-T \hat{\epsilon}_{\perp} T^{\dagger}\right)|\phi\rangle .
$$

\section{APPENDIX B: GENERIC SOLUTION FOR THE MAXWELL-SCHRÖDINGER EQUATION OF $Q$-WAVE PLATES AND $Q$ RETARDERS AND ITS OVERALL PHASE}

The Maxwell-Schrödinger equation for a $q=l$ wave plate is simply given by

$$
2 i \partial_{\delta} \psi_{ \pm, l}=e^{\mp 2 i l \bar{\alpha}} \psi_{\mp, l} .
$$

We obtain the second-order differential equation:

$$
(2 i)^{2} \partial_{\delta}^{2} \psi_{ \pm . l}=\psi_{ \pm, l} \text {. }
$$

Thus, the general solution of the Maxwell-Schrödinger equation is

$$
\psi_{ \pm, l}(\delta)=A_{ \pm+} e^{i \delta / 2}+A_{ \pm-} e^{-i \delta / 2},
$$


where $A_{ \pm \pm}$are constants. Here, we set the initial condition as follows:

$$
\psi_{ \pm, l}(\delta=0) \equiv e^{\mp i \theta_{0}} A_{ \pm}^{0} .
$$

Equation (B4) describes the relationship between $A_{ \pm \pm}$ and $A_{ \pm}^{0}$ :

$$
\begin{gathered}
\psi_{ \pm, l}(\delta=0)=A_{ \pm+}+A_{ \pm-}=+e^{\mp i l \bar{\alpha}} A_{ \pm}^{0}, \\
\left.\partial_{\delta} \psi_{ \pm, l}\right|_{\delta=0}=A_{ \pm+}-A_{ \pm-}=-e^{\mp i l \bar{\alpha}} A_{\mp}^{0} .
\end{gathered}
$$

Using Eqs. (B5) and (B6), the coefficients of Eq. (B3) are written as

$$
\begin{aligned}
& A_{ \pm+}=e^{\mp i l \bar{\alpha}} \frac{A_{ \pm}^{0}-A_{\mp}^{0}}{2}, \\
& A_{ \pm-}=e^{\mp i l \bar{\alpha}} \frac{A_{ \pm}^{0}+A_{\mp}^{0}}{2} .
\end{aligned}
$$

Consequently, the general solution is expressed by

$$
\psi_{ \pm, l}(\delta)=e^{\mp i l \bar{\alpha}}\left\{A_{ \pm}^{0} \cos \left(\frac{\delta}{2}\right)-i A_{\mp}^{0} \sin \left(\frac{\delta}{2}\right)\right\}
$$

and

$$
\begin{aligned}
|\psi\rangle & =T^{\dagger}|\phi\rangle=\frac{e^{i l^{\prime} \phi} e^{-i \delta / 2}}{\sqrt{2}} R_{l(\phi+\bar{\alpha})}\left(\begin{array}{c}
A_{+}^{0}+A_{-}^{0} \\
i\left(A_{+}^{0}-A_{-}^{0}\right) e^{i \delta}
\end{array}\right) \\
& =e^{i l^{\prime} \phi} e^{-i \delta / 2} R_{l(\phi+\bar{\alpha})}\left(\begin{array}{c}
E_{x}^{0} \\
E_{y}^{0} e^{i \delta}
\end{array}\right),
\end{aligned}
$$

where $e^{i l^{\prime} \phi} R_{l(\phi+\bar{\alpha})}\left(E_{x}^{0} \quad E_{y}^{0}\right)^{\mathrm{T}}$ is the initial state vector.

We can transform the vector $|\psi\rangle$ into the vector $\left|\psi^{\prime}\right\rangle$ whose $x$ component is real:

$$
\begin{aligned}
\left|\psi^{\prime}\right\rangle & =\exp \left[-i \Phi_{\text {overall }}\right]|\psi\rangle \\
& =\left(\begin{array}{c}
\left|E_{x}^{0} e^{-i \delta / 2} \cos \theta-E_{y}^{0} e^{i \delta / 2} \sin \theta\right| \\
\left(E_{x}^{0} e^{-i \delta / 2} \sin \theta+E_{y}^{0} e^{i \delta / 2} \cos \theta\right) \exp \left\{-i\left[l^{\prime} \phi-\arg \left(E_{x}^{0} e^{-i \delta / 2} \cos \theta-E_{y}^{0} e^{i \delta / 2} \sin \theta\right)\right]\right\}
\end{array}\right),
\end{aligned}
$$

where

$$
\Phi_{\text {overall }}=l^{\prime} \phi-\arg \left(E_{x}^{0} e^{-i \delta / 2} \cos \theta-E_{y}^{0} e^{i \delta / 2} \sin \theta\right)
$$

is an overall phase of $|\psi\rangle$ [3], and $\theta=l(\phi+\bar{\alpha})$.

Here, we show that the overall phase does not depend on the initial state. Since the overall phase is written in the form of

$$
\Phi_{\text {overall }}=\int \partial_{l}\left(\Phi_{\text {overall }}\right) d l+\text { const. }
$$

it is sufficient to show that $\partial_{l}\left(\Phi_{\text {overall }}\right)$ is independent of the initial state of

$$
e^{i l^{\prime} \phi} R_{l\left(\phi+\theta_{0}\right)}\left(\begin{array}{c}
\cos \frac{\alpha}{2} e^{-i \kappa / 2} \\
\sin \frac{\alpha}{2} e^{i \kappa / 2}
\end{array}\right)
$$

Here, $\partial_{l}\left(\Phi_{\text {overall }}\right)$ is calculated by

$$
\begin{aligned}
\partial_{l}\left(\Phi_{\text {overall }}\right) & =\partial_{l} \arg \left(E_{x}^{0} e^{-i \delta / 2} \cos \theta-E_{y}^{0} e^{i \delta / 2} \sin \theta\right) \\
& =-\partial_{l} \arctan \frac{\cos \left(\frac{\alpha}{2}-l \phi-l \bar{\alpha}\right) \sin \left(\frac{\delta+\kappa}{2}\right)}{\cos \left(\frac{\alpha}{2}+l \phi+l \bar{\alpha}\right) \cos \left(\frac{\delta+\kappa}{2}\right)} \\
& =\frac{\phi}{2} \frac{\sin \alpha \sin (\delta+\kappa)}{\cos ^{2} \frac{\alpha}{2} \cos ^{2}(l \phi+l \bar{\alpha})+\sin ^{2} \frac{\alpha}{2} \sin ^{2}(l \phi+l \bar{\alpha})-\frac{1}{2} \sin \alpha \cos (\delta+\kappa) \sin (2 l \phi+2 l \bar{\alpha})}
\end{aligned}
$$

Since the $l$ th hybrid-order Stokes parameters are

$$
\begin{gathered}
S_{1}^{l}=\cos (2 \bar{\alpha}) \cos \alpha-\sin (2 \bar{\alpha}) \sin \alpha \cos (\delta+\kappa), \\
S_{2}^{l}=\sin (2 \bar{\alpha}) \cos \alpha+\cos (2 \bar{\alpha}) \sin \alpha \cos (\delta+\kappa), \\
S_{3}^{l}=\sin \alpha \sin (\delta+\kappa),
\end{gathered}
$$

we obtain

$$
\partial_{l}\left(\Phi_{\text {overall }}\right)=\frac{S_{3}^{l} \phi}{1+S_{1}^{l} \cos (2 l \phi)-S_{2}^{l} \sin (2 l \phi)},
$$

which does not depend on the initial state. From this result, $\Phi_{\text {overall }}$ is a single-valued function of $\tilde{\boldsymbol{S}}_{l}$.
[1] J. Anandan, Nature (London) 360, 307 (1992).

[2] S. Pancharatnam, Proc. Indian Acad. Sci. A 44, 247 (1956).

[3] R. Bhandari and J. Samuel, Phys. Rev. Lett. 60, 1211 (1988); T. H. Chyba, R. Simon, L. J. Wang, and L. Mandel, Opt. Lett.
13, 562 (1988); T. D. Visser, T. van Dijk, H. F. Schouten, and W. Ubachs, Opt. Express 18, 10796 (2010).

[4] L. Allen, J. Courtial, and M. J. Padgett, Phys. Rev. E 60, 7497 (1999). 
[5] G. Milione, S. Evans, D. A. Nolan, and R. R. Alfano, Phys. Rev. Lett. 108, 190401 (2012).

[6] K. Toyoda, F. Takahashi, S. Takizawa, Y. Tokizane, K. Miyamoto, R. Morita, and T. Omatsu, Phys. Rev. Lett. 110, 143603 (2013); V. Parigi, V. D'Ambrosio, C. Arnold, L. Marrucci, F. Sciarrino, and J. Laurat, Nat. Commun. 6, 7706 (2015).

[7] C. Maurer, A. Jesacher, S. Fürhapter, S. Bernet, and M. RitschMarte, New J. Phys. 9, 78 (2007).

[8] G. Milione, H. I. Sztul, D. A. Nolan, and R. R. Alfano, Phys. Rev. Lett. 107, 053601 (2011).

[9] A. Holleczek, A. Aiello, C. Gabriel, C. Marquardt, and G. Leuchs, Opt. Express 19, 9714 (2011).

[10] X. Yi, Y. Liu, X. Ling, X. Zhou, Y. Ke, H. Luo, S. Wen, and D. Fan, Phys. Rev. A 91, 023801 (2015).

[11] G. Biener, A. Niv, V. Kleiner, and E. Hasman, Opt. Lett. 27, 1875 (2002); Z. Bomzon, G. Biener, V. Kleiner, and E. Hasman, ibid. 27, 1141 (2002).

[12] L. Marrucci, C. Manzo, and D. Paparo, Phys. Rev. Lett. 96, 163905 (2006).

[13] R. Y. Chiao and Y.-S. Wu, Phys. Rev. Lett. 57, 933 (1986).

[14] M. V. Berry, J. Mod. Opt. 34, 1401 (1987).

[15] H. Kuratsuji and S. Kakigi, Phys. Rev. Lett. 80, 1888 (1998);
R. Botet and H. Kuratsuji, Phys. Rev. E 81, 036602 (2010).

[16] M. V. Berry, Proc. R. Soc. London, Ser. A 392, 45 (1984).

[17] B. R. Holstein, Am. J. Phys. 57, 1079 (1989).

[18] Y. Aharonov and D. Bohm, Phys. Rev. 115, 485 (1959).

[19] Similar experiments can be performed by cw lasers.

[20] M. Sakamoto, K. Oka, and R. Morita, Proc. SPIE 8274, 827414 (2012).

[21] E. Karimi, G. Zito, B. Piccirillo, L. Marrucci, and E. Santamato, Opt. Lett. 32, 3053 (2007).

[22] M. Sakamoto, K. Oka, R. Morita, and N. Murakami, Opt. Lett. 38, 3661 (2013).

[23] K. C. Wright, L. S. Leslie, and N. P. Bigelow, Phys. Rev. A 77, 041601 (2008); K. C. Wright, L. S. Leslie, A. Hansen, and N. P. Bigelow, Phys. Rev. Lett. 102, 030405 (2009); J. T. Schultz, A. Hansen, and N. P. Bigelow, Opt. Lett. 39, 4271 (2014); J. T. Schultz, A. Hansen, J. D. Murphree, M. Jayaseelan, and N. P. Bigelow, J. Opt. (Bristol, UK) 18, 064009 (2016).

[24] A. Volyar and T. Fadeeva, Opt. Spectrosc. 101, 450 (2006).

[25] E. Brasselet, Y. Izdebskaya, V. Shvedov, A. S. Desyatnikov, W. Krolikowski, and Y. S. Kivshar, Opt. Lett. 34, 1021 (2009).

[26] M. Suzuki, K. Yamane, K. Oka, Y. Toda, and R. Morita, Opt. Express 22, 16903 (2014). 